\title{
Correlation between Forced Expiratory Volume in First Second (FEV1) And 6 Minute Walk Distance In Moderate, Severe and Very Severe Chronic Obstructive Pulmonary Disease
}

\author{
Anil Kumar Kodavala ${ }^{1}$, Somanath Dash ${ }^{2}$, \\ ${ }^{1,2}$ Department of pulmonology, GSL Medical college, Rajahmundry, India
}

\begin{abstract}
Relation between 6 minute walk test and spirometric parameters [FEVI and FVC] in patients with COPD is investigated. There is no published data in India in this respect. Hospital based cross sectional study. Clinical COPD questionnaire score, Forced expiratory volume in first second (FEVI) and Forced expiratory volume $(F V C), 6$ minute walk distance and 6 minute walk work were calculated and compared. Data was analyzed by SPSS 11. It was observed that $11.1 \%$ had overall clinical copd questionnaire (CCQ) score of more than 3; 62.2\% had baseline PaO2 between 60-80 mm Hg;88.9\% had baseline PaCO2 lower than or equal to 45 $\mathrm{mm} \mathrm{Hg} ; 53.3 \%$ had severe COPD according to GOLD spirometry criteria;13.3\% had complication of Pulmonary artery hypertension; $64.5 \%$ walked more than 300 meters distance and $21.1 \%$ (8) of patients with severe copd had significant desaturation during walk test. There was low positive correlation with no statistical significance between FEVI and 6 MWD $(r=0.280, p=0.062)$ and between FVC and 6 minute walk distance $(r=$ $0.289, p=0.055)$. Demographic parameters like age, BMI, smoking score, spirometry and baseline paco 2 were not correlating with 6 minute walk distance. BMI and FEVI (forced expiratory volume) were more correlating with 6 minute walk work. Severity of airway obstruction or pulmonary artery hypertension was not predicting significant desaturation during walk test. Measuring walk distance alone is not sufficient for assessment of severity and functional status of COPD the patients. 6 minute walk work, which is the product of walk distance and body weight, is more correlating with severity of the disease.
\end{abstract}

Key Words: COPD, FEVI, FVC, CCQ score, 6MWD, 6MWW.

\section{Introduction}

Chronic obstructive pulmonary disease (COPD) is a major cause of disability and death all over the world. It is the fourth and the only leading cause of death showing increase in prevalence worldwide. In India, it was reported a median COPD prevalence of 5 percent in males and 2.7 percent in females in adults of 30 years age and above. The 5-year mortality rate for patients with COPD varies from $40 \%$ to $70 \%$. Several factors, including forced expiratory volume in 1 second (FEV1), airway hyper-responsiveness, severity of dyspnea, gas exchange disturbances, lung hyperinflation, pulmonary hypertension, malnutrition-impaired exercise capacity and health-related quality of life, anemia, and other co morbidities have been identified as individual predictors of mortality in COPD. Spirometry is the present gold standard for diagnosing COPD. FEV1/FVC ratio is generally used to define the presence or absence of airflow limitation, but FEV1 is used to define the severity of the disease. FEV1 correlates weakly with dyspnea, accounting for only $12 \%$ of the variability in dyspnea as measured by the Baseline Dyspnea Index (BDI). Once FEV1 is reduced to 35\% of predicted, there is a wide spectrum of ability; some patients are not very limited in their activities, whereas others are confined to wheelchairs. This enigma points to the need for other assessment tools.

6-min walk distance (6MWD) test is a sub maximal exercise test, used to assess the functional status of patients with COPD. 6MWD test had proved to be reliable, inexpensive, safe and easy to apply. It evaluates global and integrated response of all systems involved during exercise like pulmonary, cardiovascular, musculoskeletal and neuromuscular. As spirometry depends on effort, all patients may not be comfortable in doing the test. Patients with very severe COPD, based on FEV1, have variable exercising ability. On the other hand 6MWD test is easy to perform as it is representing daily activity. If there is correlation between 6MWD and FEV1, 6MWD test can be used for assessing the severity of COPD where spirometry can not be done. There is no published data in India in this respect. In this study, the relation between 6MWD test and spirometric parameters [FEV1 and FVC] in patients with COPD is investigated.

\section{Materials And Methods}

In this hospital-based cross sectional study, 45 patients were included in the study with history of smoking, clinical features of persistent, progressive and exertional dyspnea, chronic cough with sputum and radiological examination suggestive of COPD. According to GOLD criteria, diagnosis was confirmed by using 
FEV1/FVC, FEV1\% predicted and patients with moderate, severe and very severe disease with the following features were selected.

The following patients were excluded from the study

1. COPD patients with acute exacerbations within last 6 weeks

2. Post bronchodilator reversibility $>12 \%$ and $>200 \mathrm{ml}$

3. Patients receiving domiciliary oxygen therapy.

4. Patients who could not perform spirometry or walk test.

5. Patients with other diseases affecting respiratory and cardiovascular systems

6. Recent thoracic or abdominal surgery/eye surgery or retinal detachment.

7. Patients who are musculoskeletal pain limiting, claudication pain and syncope.

Informed consent was taken from all subjects and then they were interviewed, demographic characteristics, clinical history was noted, physical examination was done, quality of life was assessed by using CCQ score, investigations including chest X-ray, ABG, ECG, spirometry, 2-D Echo were done and and then they were instructed to do 6 minute walk test.

Using spirometer (Micro Quark, Cosmed, Rome, Italy) FEV1/FVC, FEV1, FVC, FEV1 \% predicted, $\mathrm{FVC} \%$ predicted, $\mathrm{FEF}_{25-75 \%}$ were obtained before and after the patient received salbutamol via a metered-dose inhaler. All patients had prior instruction in the use of metered-dose inhalers and were observed for the effective administration of the bronchodilator. The protocol was to administer salbutamol (100mcg/puff) 2 puffs, wait for $20 \mathrm{~min}$ and then to do post bronchodilator spirometry. Pulmonary function testing was performed and severity was defined according to the GOLD guidelines. The best flow-volume loop was used in the final analysis of the data. All calculations were performed using automated processors that were interfaced to the measuring instruments. Severity of disease was assessed by FEV1 \% predicted according to GOLD criteria.

A 100 merter straight hospital corridor with an even walking surface was used and was marked by colored tape at each end. Before starting the walk, a PFT technician recorded dyspnea, as measured with the modified Borg dyspnea scale, pulse rate and oxygen saturation ( $\mathrm{SaO} 2)$. Patients were instructed to walk end to end at theirs own pace, while attempting to cover as much distance as possible in the allotted 6 min. The PFT technician timed the walk and offered verbal encouragement to each patient. Patients were allowed to stop and rest as necessary but were encouraged to proceed with the walk on recovery. At the end of 6 minutes, the total distance covered was recorded to the nearest meter. Dyspnea scale, oxygen saturation and pulse rate were also recorded after the test. $6 \mathrm{MWW}$ was calculated as the product of walk distance and body weight. ${ }^{1}$

Using echo Right Ventricular Systolic Pressure (RVSP) was assessed. RVSP $\geq 30+$ RAP was considered as presence of pulmonary artery hypertension.

\section{Data Analysis}

Data of was analyzed by SPSS 11 (the Statistical Program for the Social Sciences of the University of Michigan, Microsoft Corporation, Redmond, USA). Continuous variables were expressed as mean \pm standard deviation (SD), and categorical variables were expressed in numbers and percentages. Descriptive measures were taken for continuous variables like age, BMI, smoking score, CCQS, FEV1, FVC, $\mathrm{PaO} 2, \mathrm{PaCo} 2, \mathrm{SpO} 2$, 6MWD and 6MWW. Pearson's product moment correlation was used to find correlation between FEV1 and 6MWD (6Minute Walk Distance). Chi-square technique was used to find the association between categorical variables and the outcome (Exercise capacity and significant desideration). A 'p' value of less than 0.05 was taken as significant.

\section{Results}

In our study ( $\mathrm{n}=45$ ), all patients were males. All patients were of more than 50yrs with a mean age of $65.67 \pm 7.087$ years. 40 patients $(88.9 \%)$ were from middle socioeconomic class. The mean body mass index (BMI) was $21.67 \pm 3.65 ; 11$ patients $(24.5 \%)$ had over weight and 10 patients $(22.2 \%)$ had under weight. 24 patients $(53.3 \%)$ had normal BMI. 18 patients $(40 \%)$ had smoking score of $\leq 20$ pack years and $27(60 \%)$ patients had a score of $>20$ pack years.

All patients (100\%) had shortness of breath, $91.1 \%$ patients had cough, $66.6 \%$ patients had sputum production and only $4 \%$ had wheeze at the time of presentation. $49 \%$ patients had systemic hypertension, $29 \%$ have more than one co morbid illness and nearly $27 \%$ had diabetes mellitus.

40 patients $(88.9 \%)$ had a clinical copd questionnaire (CCQ) score of $\leq 2.5$. 28 patients $(62.2 \%)$ had baseline $\mathrm{PaO} 2$ between $60-80 \mathrm{~mm} \mathrm{Hg}$ at rest. Mean $\mathrm{PaCO} 2$ is $37.17 \pm 5.55$. Only $5(11.1 \%)$ patients had hypercapnia at rest. Mean forced expiratory volume in first second (FEV1) was $0.9153 \pm 0.31228$ liters. $7(15.6 \%)$ patients had moderate COPD, 24 (53.3\%) patients had severe COPD, and 14 (31.1\%) patients had very severe 
COPD according to GOLD spirometry criteria. 6 patients (13.3\%) had complication of Pulmonary artery hypertension. Mean walking distance of study group was $333.11 \pm 82.015$ meters. 41 patients $(91.1 \%)$ have walked > 200 meters and $64.5 \%$ walked more than 300 meters distance. A drop in saturation $\geq 4 \%{ }^{2}$ from baseline was considered as significant desaturation. Total 9 patients had significant desaturation. Out of them, 8 (21.1\%) patients had severe COPD (FEV1 <50\%).

Demographic parameters like age, BMI, smoking score, spirometry and baseline paco2 were not correlating with 6 minute walk distance. BMI and FEV1 (forced expiratory volume) were more correlating with 6 minute walk work. Severity of airway obstruction or pulmonary artery hypertension was not predicting significant desaturation during walk test. TABLE 2.

Table1. Baseline patient characteristics

\begin{tabular}{|l|l|l|l|}
\hline Variable & Mean & Range & Std. Deviation \\
\hline Age(years) & 65.67 & $51-82$ & 7.087 \\
\hline $\begin{array}{l}\text { Smoking score } \\
\text { (pack years) }\end{array}$ & 32.03 & $5-200$ & 31.381 \\
\hline CCQ score & 2.556 & $1.6-4.0$ & 0.4585 \\
\hline BMI & 21.674 & $14.3-31.8$ & 3.6582 \\
\hline FEV1(liter/sec) & 0.9153 & $0.34-1.91$ & 0.31228 \\
\hline FVC(liter/sec) & 1.6813 & $0.78-2.95$ & 0.44957 \\
\hline 6MWD(meters) & 333.11 & $120-540$ & 82.015 \\
\hline 6MWW(kg-meter) & 19087.40 & $5940-38610$ & 6424.730 \\
\hline PaO2(mm Hg) & 75.584 & $58.1-93.7$ & 9.4194 \\
\hline PaCO2(mm Hg) & 37.176 & $22.1-50.0$ & 5.5536 \\
\hline Pre SPO2 & 96.40 & $92-99$ & 1.671 \\
\hline
\end{tabular}

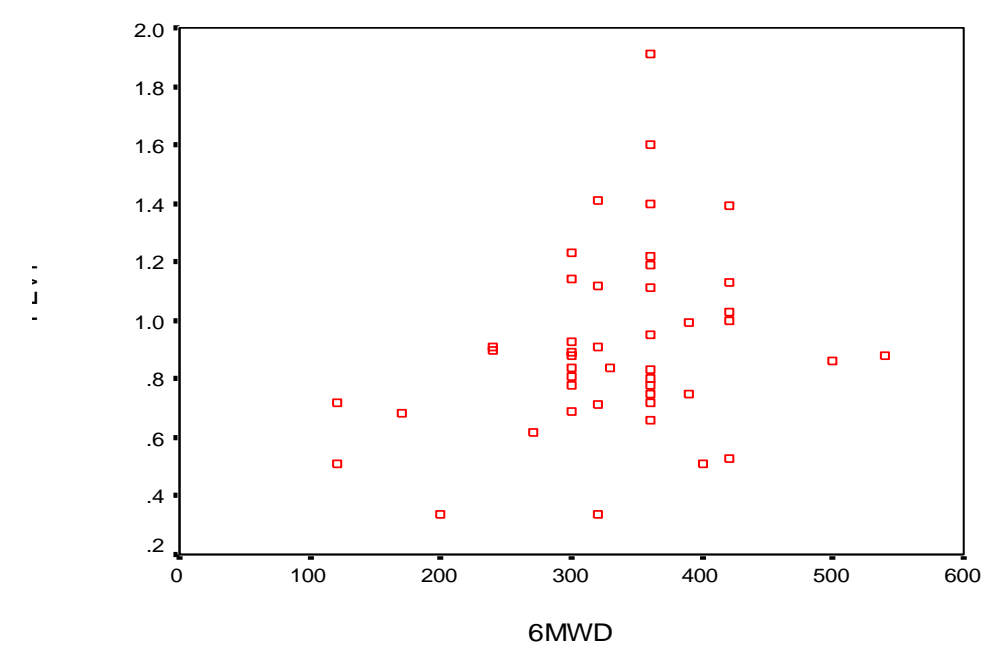

Figure 1. Graph showing the correlation between FEV1 and 6MWD

Table 2. Correlation Coefficients of 6-Minute Walking Distance and 6-minute work with Lung Function and Other Parameters

\begin{tabular}{|l|l|l|l|l|}
\hline \multirow{2}{*}{ Variable } & \multicolumn{3}{|c|}{ 6MWD } & \multicolumn{2}{l|}{ 6MWW } \\
\cline { 2 - 5 } & 'r'value & 'p' value & 'r' value & 'p' value \\
\hline Age & -0.025 & 0.872 & -0.077 & 0.615 \\
\hline BMI & 0.049 & 0.747 & 0.477 & 0.001 \\
\hline Smoking score & -0.052 & 0.736 & 0.046 & 0.764 \\
\hline CCQ score & -0.307 & 0.040 & -0.207 & 0.172 \\
\hline FEV1 & 0.280 & 0.062 & 0.370 & 0.012 \\
\hline FVC & 0.289 & 0.055 & 0.269 & 0.074 \\
\hline $\mathrm{PaCO} 2$ & -0.047 & 0.759 & 0.011 & 0.943 \\
\hline $\mathrm{PaO} 2$ & 0.086 & 0.576 & 0.203 & 0.182 \\
\hline $\mathrm{SpO} 2$ & -0.009 & 0.952 & -0.075 & 0.622 \\
\hline
\end{tabular}

* Correlation is significant at the 0.05 levels. 


\section{Discussion}

There are limited studies in this aspect and the correlation of the distance walked with spirometry lacks agreement, with some studies like Mak, VHF et al (1993). P.J.Wijkstra ${ }^{3}$ et al (1994), finding strong relationships and others like Bernstein $\mathrm{ML}^{4}$ (1994) and Elie Fiss ${ }^{5}$ et al (2006) finding weak relationships.

Usually COPD manifests after 40years of age. Our study consists of patients with an age of 50years and above. More than $51 \%$ of patients of the study belong to the age group 66-75yers. There was no significant correlation between 6minute walk distance (6MWD) and age $(r=-0.025, \mathrm{p}=0.872)$ in our study. This is similar to a study by Ozalevli et $\mathrm{al}^{6}$ in 2007, $(\mathrm{r}=-0.17)$. But in a study by Yi-Hsi Wang et al $(\mathrm{n}=60)$, in $2005,{ }^{7} 6 \mathrm{MWD}$ was correlating inversely with age $(r=-0.326, p<0.05)$. This indicates variable influence of age over 6MWD. In our study quality of life was assessed by using clinical COPD questionnaire (CCQ) score that was developed and validated by, van der Molen et $\mathbf{a l}^{\mathbf{8}}$, in 2003. CCQ score is a practical clinical instrument to be used for routine evaluation of clinical control (symptom, functional state and mental state) concerning patients with COPD, in general practice. In this study CCQ score was negatively correlating with 6MWD $(r=-0.307$, $\mathrm{p}=0.040$ ). In fact, CCQ score correlated more than FEV1 with 6MWD. This points the importance of assessing quality of life in COPD patients. Health related quality of life quantifies the impact of the disease on activities of daily living (ADLs) and the individual's sense of well-being.

Spirometry with measurements derived from a maximal forced expiratory maneuver is the most common test that is used to diagnose COPD. FEV1 has traditionally been used to grade severity of COPD. We also used spirometry for staging of COPD patients. $38(84.5 \%)$ patients had severe to very severe disease. 7(15.5\%) patients had moderate disease. 6MWD was not correlating with FEV1 ( $r=0.280, p=0.062)$ and FVC $(\mathrm{r}=0.289, \mathrm{p}=0.055)$ in our study. But in a study by Jose M. Marin et $\mathrm{al}^{9}$, in 2001, the mean 6MWD correlated positively with FVC and FEV1. Correlating mean value of walk distance with FEV1 may not represent the true relation between them as the same level of obstruction in different individuals may manifest with different functional status. In a study by Yi-Hsi Wang et $\mathrm{al}^{7}(\mathrm{n}=60)$, in 2005, they found correlation between 6MWD and forced expiratory volume in 1 second (0.258), forced vital capacity (0.273). Even though there was a positive correlation, it was not statistically significant as per values mentioned In a study by Elie Fiss et $\mathrm{al}^{5}$, in 2006, significant correlation was found between $6 \mathrm{MWD}$, absolute mean $( \pm \mathrm{SD})$ values and mean $( \pm \mathrm{SD})$ percent of predicted for VC and FVC, but not for FEV1 and inspiratory capacity. Our results were also similar to this, but we did not measure inspiratory capacity. In a study by Hatem FS Al Ameri et $a^{10}$, in 2006, they found correlation of 6 minute walk distance with DLCO, FVC and FEV1. Among these three, DLCO was strongly correlating, followed by FVC and FEV1 respectively. This study included not only COPD patients but also other respiratory disorders. But diffusing capacity was not checked in our study, as it was costly and usually not recommended for COPD patients. Our intention was to show that the 6-minute walking test is easily performed and reflects COPD patients' lung function, and that sophisticated examinations, such as diffusing capacity, may be omitted.

In a study by Tarek Safwat et $\mathrm{al}^{11}(\mathrm{n}=30)$, in 2009 , they found highly statistically significant direct relation between 6 MWD and FEV1\% predicted(r=0.77, $\mathrm{p}<0.01)$. But in our study we correlated 6 MWD with absolute value of FEV1 and not with FEV1\% predicted. At severe level of airway obstruction, absolute value of FEV1 is more reliable than $\%$ predicted. In contrast to this, a study by Ozalevli et al ${ }^{6}$ in 2007 , showed no significant relation between 6MWD and FVC \% predicted ( $\mathrm{r}=0.32)$, FEV1 \% predicted $(\mathrm{r}=0.33)$. It has been found that FEV1 impairment and perceived breathlessness are correlated with waking distance in patients with COPD. ${ }^{6}$ In general there is considerable variability in the relationships between the FEV1 and other physiological abnormalities in COPD. Again, in a study by Osamu Nishiyama et $\mathrm{al}^{12}$, in 2007, found significant correlation between 6MWD and FEV1 $(r=0.70, \mathrm{P}<0.0001)$. This shows the existence of conflicting evidence for correlation between 6MWD and FEV1 in COPD patients.

Walk work, which is the product of walk distance and body weight, was used in our study to find relation with FEV1. 6MWW rather than 6MWD was more correlating with FEV1 $(r=0.370, p=0.012)$ and $\operatorname{BMI}(r=0.477, \mathrm{p}=0.001)$. Similar finding was noted shown by Carter et $\mathrm{al}^{1}(2003)$. In that study both FEV1 and FVC were more correlating with $6 \mathrm{MWW}$ rather than $6 \mathrm{MWD}$. Our findings indicate an advantage for using the calculation of work over simple distance covered in 6-min of level walking for patients with COPD. The work calculation takes into account the mass of the body and the energy required to move that body through space. Since all individuals vary with respect to body weight, the expected work of walking/ambulation will vary. By including the work calculation for the 6MW, a major source of variation is accounted for (i.e., less scatter). Thus, the resultant measurement is considered to be more precise.

In this cross sectional study, we assessed the relation between 6 minute walk distance with forced expiratory volume (FEV1) in COPD patients of moderate to very severe stages as graded by GOLD. We want to know the importance of walking test in assessing the severity of COPD as it is a safe, simple, reliable tool and is representing routine activity of patients, when compared to spirometry. We found no significant correlation between 6minute walk distance and forced expiratory volume (FEV1). 6minute walk distance is more 
correlating with quality of life (CCQ) score and forced vital capacity (FVC). Measuring distance alone during walk test may not represent the severity of the disease and functional status of the subjects. FEV1 and BMI were more correlating with 6 minute walk work rather than 6minute walk distance. $6 \mathrm{MWW}$ may be more precise in assessing functional status and severity of the disease. Assessment of 6MWW should also be done along with 6MWD during walk test. Walking distance and occurrence of significant desaturation during walk test could not be predicted by severity of obstruction during spirometry or vice versa.

\section{Limitations}

Study sample size is 45 , results cannot be generalized and larger studies are needed. All patients in our study are males. So, findings may not be applicable to female gender. Patients with $\mathrm{PaCO}>50 \mathrm{~mm} \mathrm{Hg}$ were not included in the study. This might have lead to exclusion of some of very severe copd patients, which in turn is responsible for lack of correlation between blood gases, and walk test. Patients with resting oxygen saturation $<90 \%$ were excluded from study, this might have decreased number of patients with desaturation during walk test. 2D echo, being a noninvasive method, was used to detect pulmonary artery hypertension. Because of hyperinflation and poor echo window there was a chance of missing mild pulmonary artery hypertension. We tried to find a relation between presence or absence of pulmonary artery hypertension rather than severity of pulmonary artery hypertension and presence or absence of significant desaturation during walk test.

In conclusion, measuring 6 minute walk distance alone is not sufficient for assessment of severity of COPD and functional status of the patients. 6 minute walk work, which is the product of walk distance and body weight, is more correlating with severity of the disease. In case of unavailability of spirometry due to limited resources, $6 \mathrm{MWW}$ is a reasonable predictor for assessing severity and functional status of COPD patients. Further randomized controlled studies are required in promoting 6minute walk work for assessing severity of COPD.

\section{Acknowledgements}

The authors thank Dr Hari Lakshmanan, Dr Darsana viswam, Dr Kumari Indira for their valuable suggestions and overall guidance in conducting this study and preparation of the manuscript.

\section{References}

[1] Carter R, Holiday DB, Nwasuruba C, Stocks J, Grothues C, Tiep B. 6-minute walk work for assessment of functional capacity in patients with COPD. Chest 2003;123:1408-15

[2] Casanova C, Cote C, Marin JM, Pinto-Plata V, de Torres JP, Aguirre-Jaime A, et al. Distance and oxygen desaturation during the 6min walk test as predictors of long-term mortality in patients with COPD. Chest 2008; 134:746-52.

[3] Wijkstra PJ, TenVergert EM, van der Mark TW, Postma DS, Van Altena R, Kraan J, et al. Relation of lung function, maximal Inspiratory pressure, dyspnoea, and quality of life with exercise capacity in patients with chronic obstructive pulmonary disease. Thorax 1994; 49: 468-72.

[4] Bernstein ML, Despars JA, Singh NP, Avalos K, Stansbury DW, Light RW. Reanalysis of the 12-min walk in patients with chronic obstructive pulmonary disease. Chest 1994; 105: 163-7.

[5] Elie Fiss. Six minute walk test and spirometric parameter correlations in the chronic obstructive pulmonary disease patient. Chest 2006; 130:174S-175S.

[6] Ozalevli S, Ozden A, Gocen Z, Cimrin AH. Comparision of six minute walking tests conducted with and without supplemental oxygen in patients with chronic obstructive pulmonary disease and exercise-induced oxygen desaturation. Ann Saudi Med 2007; 27:94-100.

[7] Woon WT, Fang WF, Lin MC, Wang YH. Six-Minute Walking Test in Patients with Chronic Obstructive Pulmonary Disease. Thorac Med 2005; 20:431-7.

[8] 8.van der Molen T, Willemse BW, Schokker S, ten Hacken NH, Postma DS, Juniper EF. Development, validity and responsiveness of the Clinical COPD Questionnaire. Health Qual Life Outcomes 2003; 1:13

[9] Marin JM, Carrizo SJ, Gascon M, Sanchez A, Gallego B, Celli BR. Inspiratory capacity, dynamic hyperinflation, breathlessness, and exercise performance during the 6-minute-walk test in chronic obstructive pulmonary disease. Am J Respir Crit Care Med 2001; 163:1395-9.

[10] Al Ameri HFS. 6 minute walk test in respiratory diseases: a university hospital experience. Ann Thorac Med 2006; 1: 16-19.

[11] Tarek Safwat, Khaled Wagih, Dina Fathy. Correlation between forced expiratory volume in the first second (FEV1) and diffusion capacity of the lung for carbon monoxide (DLCO) in chronic obstructive pulmonary disease. EJB 2009; 3:119-123.

[12] Nishiyama O, Taniguchi H, Kondoh Y, Kimura T, Kato K, Ogawa T, et al. Dyspnoea at 6-min walk test in idiopathic pulmonary fibrosis:comparison with COPD. Respir Med 2007; 101:833-8. 